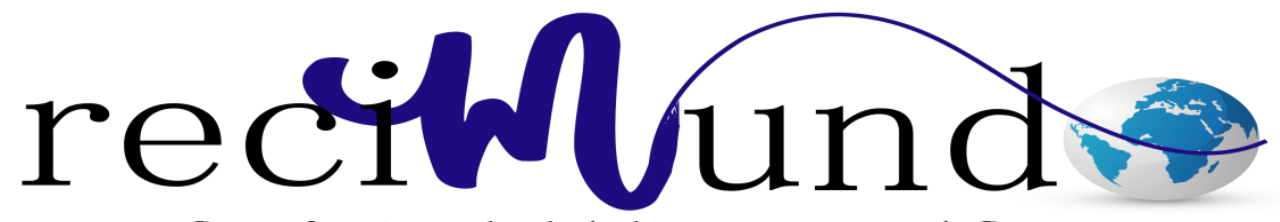

Revista Científica Mundo de la Investigación y el Conocimiento

Thalía Carolina Salgado Rosado a , Juan Carlos Obando Piedra b, Pablo Rafael Salgado Rosado ${ }^{c}$, Walter Kiko Salgado ${ }^{d}$

Tratamiento quirúrgico de la anquiloglosia recidivante: A propósito de un caso

Revista Científica Mundo de la Investigación y el Conocimiento. Vol. 1 núm., 4, septiembre, 2017, pp. 777-790

DOI: $10.26820 /$ recimundo/1.4.2017.777-790

Editorial Saberes del Conocimiento 


\section{Tratamiento quirúrgico de la anquiloglosia recidivante: A propósito de un caso \\ Vol. 1, núm. 4., (2017) \\ Thalía Carolina Salgado Rosado, Juan Carlos Obando Piedra, Pablo Rafael Salgado Rosado, Walter Kiko Salgado}

\section{RESUMEN}

La anquiloglosia es una anomalía congénita determinada por un frenillo lingual anormalmente corto, que origina dificultades en grados variables de la movilidad lingual; Reportamos el caso de un paciente masculino de 17 años de edad, con frenillo lingual recidivante con problemas en la articulación de palabras, antecedente quirúrgico de frenotomía en sus primeros años de vida para la corrección de la anquiloglosia, esta intervención no tuvo el resultado esperado, ya que se mejoró la lactancia materna, pero el paciente en la actualidad presenta una anquiloglosia grado 3 con movilidad moderada de la lengua y dificultad para la articulación de las palabras . El paciente fue tratado basándonos en el protocolo rehabilitador-quirúrgico del servicio de cirugía maxilofacial y el servicio de terapia de lenguaje del Hospital de Nens de Barcelona; Para la intervención quirúrgica ejecutamos una frenectomía lingual con una sola pinza hemostática. El paciente obtuvo un excelente resultado optimizando la movilidad lingual, y por consiguiente mejoro la articulación de las palabras. Concluimos que la técnica quirúrgica de frenectomía acompañada de una rehabilitación de ejercicios miofuncionales antes y después de la cirugía, obtuvo resultados aceptables.

Palabras Claves: Frenillo lingual; tratamiento quirúrgico; anquiloglosia. 


\title{
Tratamiento quirúrgico de la anquiloglosia recidivante: A propósito de un
}

Vol. 1, núm. 4., (2017)

Thalía Carolina Salgado Rosado, Juan Carlos Obando Piedra, Pablo Rafael Salgado Rosado, Walter Kiko Salgado

\begin{abstract}
Ankyloglossia is a congenital anomaly characterized by an abnormally short lingual frenulum resulting in varying degrees of difficulty of tongue mobility. We describe a case of a 17 years old male patient with recurrent lingual frenulum and speech problems. He has a surgical intervention when he was a toddler, this surgical procedure did not get the result expected because he did improve his breastfeeding problem but he still has degree three of Ankyloglossia with moderate mobility. The patient was treated base in a protocol of the Service of Maxillofacial Surgery and the Service of Speech Therapy of pediatric Hospital of Nens, Barcelona ; which consist in lingual myofunctional rehabilitation before and after the surgery. The technical used was frenectomy with one haemostatic. The patient obtained a great result improving his tongue mobility, and therefore he got a major improve in speech. That shows that the frenectomy procedure associate with Myofunctional rehabilitation before and after the surgery will get great results.
\end{abstract}

Keywords: Ankyloglossia; lingual frenulum; surgery treatment. 


\section{Tratamiento quirúrgico de la anquiloglosia recidivante: A propósito de un caso \\ Vol. 1, núm. 4., (2017) \\ Thalía Carolina Salgado Rosado, Juan Carlos Obando Piedra, Pablo Rafael Salgado Rosado, Walter Kiko Salgado}

\section{Introducción.}

La Anquiloglosia es definida como una limitación de las posibilidades de protrusión y elevación de la punta de la lengua debido a un frenillo lingual corto o al musculo geniogloso corto o ambos; debido a esto, la anquiloglosia está asociada a disfunciones en la lactancia materna y en la articulación de palabras, problemas ortodonticos y sociales que afectan al paciente. Puede aparecer como una anomalía aislada, aunque a veces puede estar acompañada por otras anomalías congénitas como paladar hendido. Tiene una naturaleza hereditaria y ocurre más comúnmente en niños varones, y ha sido relacionado al cromosoma sexual X. Existen diferentes técnicas quirúrgicas para la remoción de un frenillo lingual; frenotomía, frenectomía y la frenuloplastia; no existe un parámetro concluyente que limite cuando es necesario realizar una pero es concluyente saber que es necesaria la cirugía antes que el infante desarrolle una alteración en el habla y problemas de alimentación. ${ }^{1-3}$

El servicio de cirugía maxilofacial y el servicio de terapia de lenguaje del Hospital de Nens de Barcelona realizo un protocolo exclusivo para la correcta rehabilitación y el éxito del tratamiento de la anquiloglosia en la infancia y la adolescencia; publicado en el "Journal section: Oral Medicine and patology”, 2016. Este protocolo empieza con ejercicios de terapia de lenguaje una semana antes de la cirugía, para que el paciente aprenda la realización de los ejercicios sin dolor. Los ejercicios se repiten 24 horas después de la cirugía (2 veces) y a las 48 horas (3 veces). Se debe realizar controles a las 72 horas, a las 15 y a los 45 días para evaluar la realización de los ejercicios, el tono y el desarrollo motor de la musculatura lingual. ${ }^{4-7}$ 


\section{Tratamiento quirúrgico de la anquiloglosia recidivante: A propósito de un}

Vol. 1, núm. 4., (2017)

Thalía Carolina Salgado Rosado, Juan Carlos Obando Piedra, Pablo Rafael Salgado Rosado, Walter Kiko Salgado

\section{Reporte del Caso}

Paciente masculino de 17 años de edad, acude a la Clínica integral de la Facultad Piloto de Odontología de la Universidad de Guayaquil por la preocupación de sus padres, manifestando que su hijo tiene "Problemas de pronunciación debido a su frenillo lingual". Al interrogatorio con los padres refieren que fue realizada una intervención quirúrgica para remover el frenillo lingual a los 13 meses de vida en el "Hospital Alejandro Man", lo cual mejoro el problema en la lactancia materna, pero no tuvo el éxito terapéutico en la dislalia, aun cuando se realizó terapia de lenguaje por un año.

Mediante el diagnóstico completo Intraoral encontramos un paciente con dentición permanente, sus arcadas: maxilar en forma oval y mandíbula en forma cuadrada, presencia de Torus mandibular, ligero apiñamiento en el sector anterior inferior, lengua de tamaño normal, inserción del frenillo lingual aberrante anterior, paladar duro y blando normal, encías no presentan alteración de forma, tamaño y color y sus amígdalas se encuentran sin inflamación. ${ }^{8-10}$

Se realizaron las pruebas para comprobar la presencia de anquiloglosia basándonos en la prueba del servicio de cirugía maxilofacial y el servicio de terapia de lenguaje del Hospital de Nens de Barcelona; el cual consiste en clasificar la anquiloglosia según el grado de movilidad de la lengua ; Cuando le solicitamos al paciente que contacte la punta de la lengua con el paladar, se observa que la lengua ocupa la mitad del espacio intraoral (IMAGEN 1) y la tensión del frenillo produce una leve apariencia de corazón, es así que comprobamos que el paciente presenta grado 3 de anquiloglosia con moderado movimiento lingual. ${ }^{9}$ 
Tratamiento quirúrgico de la anquiloglosia recidivante: A propósito de un caso

Vol. 1, núm. 4., (2017)

Thalía Carolina Salgado Rosado, Juan Carlos Obando Piedra, Pablo Rafael Salgado Rosado, Walter Kiko Salgado

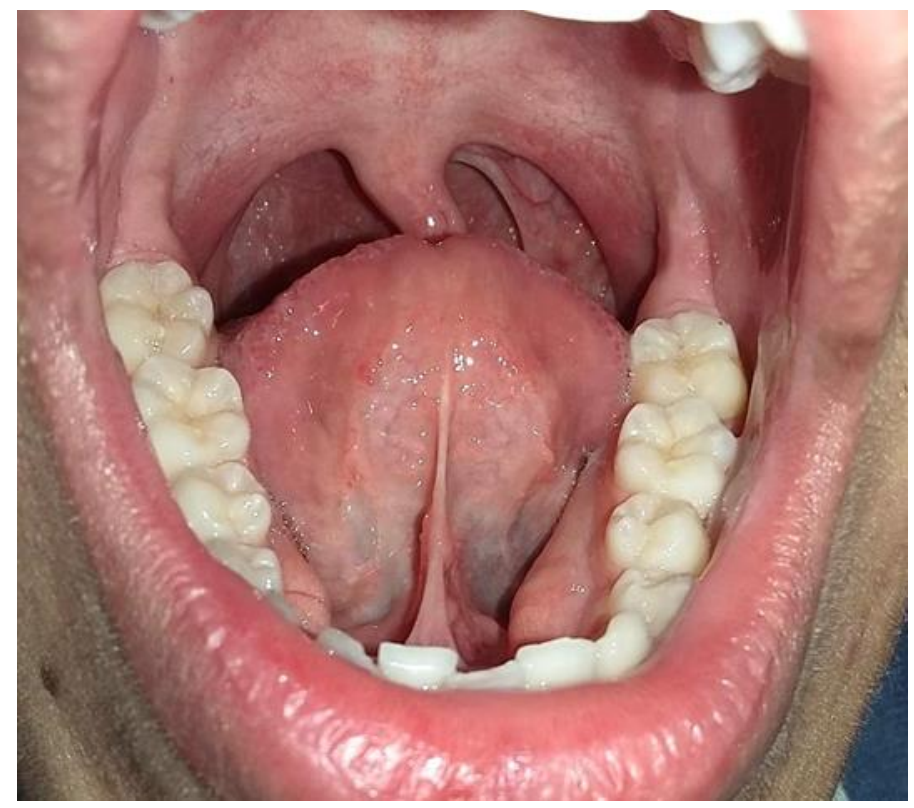

Imagen 1 Prueba Para Comprobar La Presencia De Frenillo Lingual. Fuente: Registro De La Investigación Autor: Thalía Salgado Rosado

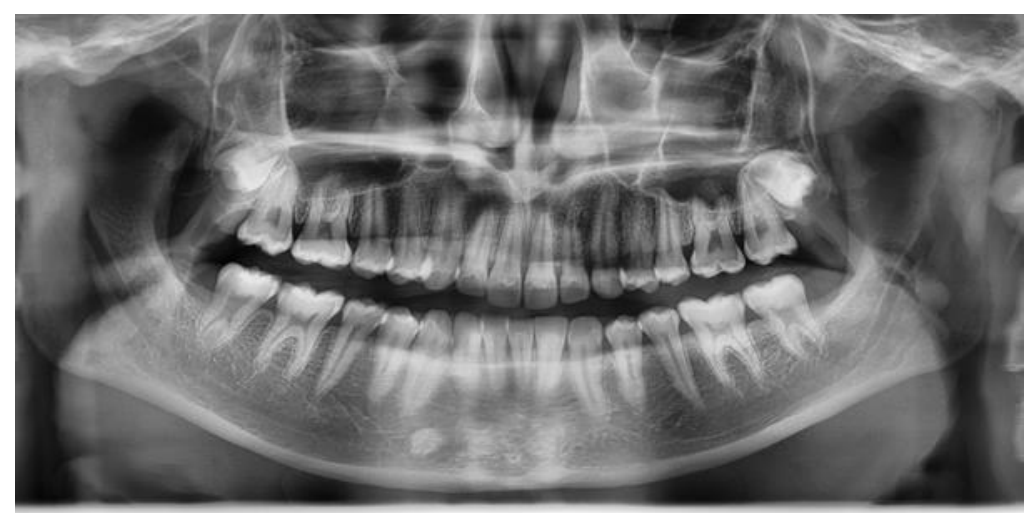

Imagen 2 Radiografía Panorámica Fuente: Registro De La Investigación Autor: Thalía Salgado Rosado 


\section{Tratamiento quirúrgico de la anquiloglosia recidivante: A propósito de un}

caso

Vol. 1, núm. 4., (2017)

Thalía Carolina Salgado Rosado, Juan Carlos Obando Piedra, Pablo Rafael Salgado Rosado, Walter Kiko Salgado

Se ordenó una radiografía panorámica ya que es de vital importancia para observar el estado general de la boca y sus estructuras vecinas. El paciente presenta: Dientes \#18 y \#28 en evolución normal intraóseo, Anodoncia de \#38 y \#48 y Taurodoncia \#37 y \#47. (IMAGEN 2)

\section{Tratamiento quirúrgico}

Como tratamiento definitivo se eligió frenectomía lingual, después de analizar la historia clínica y los exámenes de laboratorio del paciente, se concluyó que esta es la mejor técnica quirúrgica aplicable al frenillo lingual recidivante (grado 3 en movilidad) del paciente para corregir su dislalia. Explicamos al paciente el protocolo de tratamiento a realizarle y acordó colaborar con los ejercicios de rehabilitación miofuncionales antes de la intervención. ${ }^{10}$

Se realizó una técnica anestésica infiltrativa a ambos lados del frenillo y en todo su recorrido, utilizando una aguja corta e infiltrando con lidocaína al $2 \%$ con vasoconstrictor (1:800,000 epinefrina). (IMAGEN 3)

Para la preparación de la zona operatoria procedimos a elevar la lengua con un punto de sutura utilizando Hilo de seda 0.3 para la tracción en la punta lingual. Al traccionar de este punto logramos levantar con facilidad la lengua y obtener una buena visibilidad y control de la lengua para proceder a la incisión. ${ }^{11-13}$ 


\section{Tratamiento quirúrgico de la anquiloglosia recidivante: A propósito de un}

caso

Vol. 1, núm. 4., (2017)

Thalía Carolina Salgado Rosado, Juan Carlos Obando Piedra, Pablo Rafael Salgado Rosado, Walter Kiko Salgado

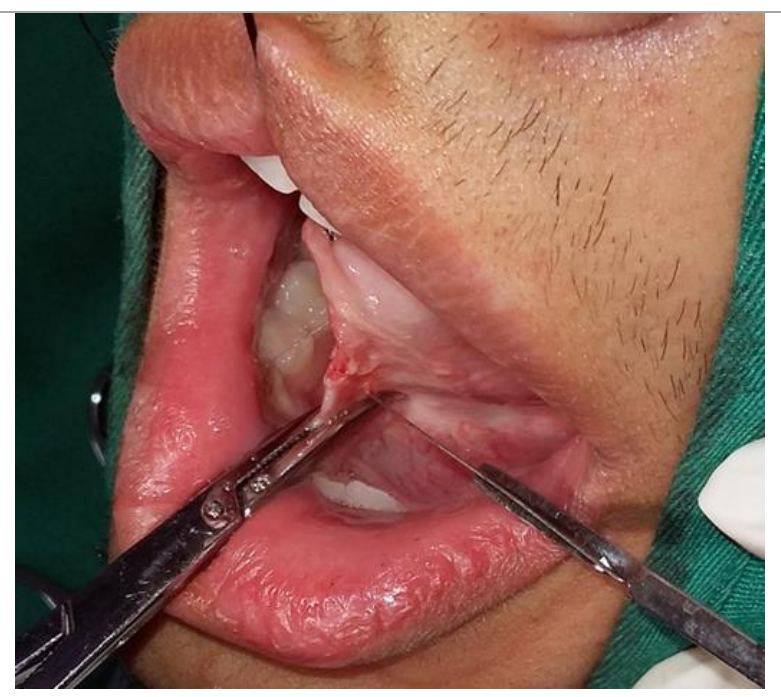

\section{Imagen 3.- Incisión}

Fuente: Registro De La Investigación

Autor: Thalía Salgado Rosado

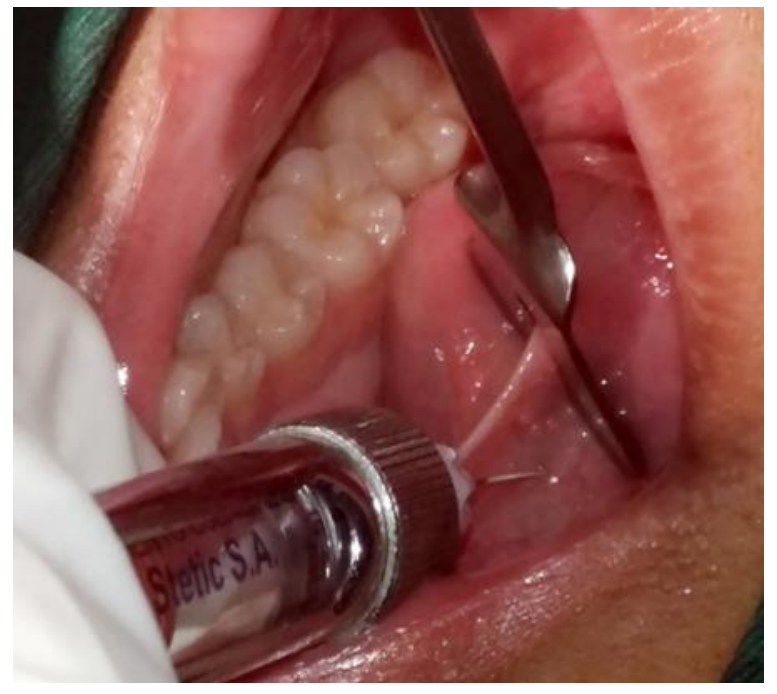

Imagen 4 anestesia infiltrativa

Fuente: registro de la investigación

Autor: thalía salgado rosado

Escogimos la técnica de frenectomía con un solo hemostático, debido a que el frenillo era muy corto y no había espacio suficiente para colocar dos hemostáticos; Primero clampeamos el 


\title{
Tratamiento quirúrgico de la anquiloglosia recidivante: A propósito de un
}

caso

Vol. 1, núm. 4., (2017)

Thalía Carolina Salgado Rosado, Juan Carlos Obando Piedra, Pablo Rafael Salgado Rosado, Walter Kiko Salgado

frenillo con una sola pinza hemostática curva entre la cara ventral de la lengua y los carúnculas de wharton, teniendo siempre presente localizarnos a $1 \mathrm{~cm}$ de los carúnculas de wharton, para evitar lesionarlos. (IMAGEN 4). Utilice una hoja de bisturí \#15 siguiendo la curvatura de la pinza hemostática, cortando la parte más alto del frenillo. La segunda incisión la hice en la parte más baja del frenillo, cerca del piso de la boca. Después de hacer la extirpación completa del tejido remante del frenillo lingual, procedimos a hacer la liberación de las fibras musculares (geniogloso); esto lo realizamos seccionado suavemente y transversalmente con la punta roma de la pinza hemostática curva el musculo geniogloso, hasta que obtuvimos el grado de movilidad que deseábamos. (IMAGEN 5) Esta fase la comprobamos haciendo que el paciente trate de contactar la punta de la lengua con el paladar; al ver que nuestro paciente dio positivo a la prueba; procedimos con la sutura.

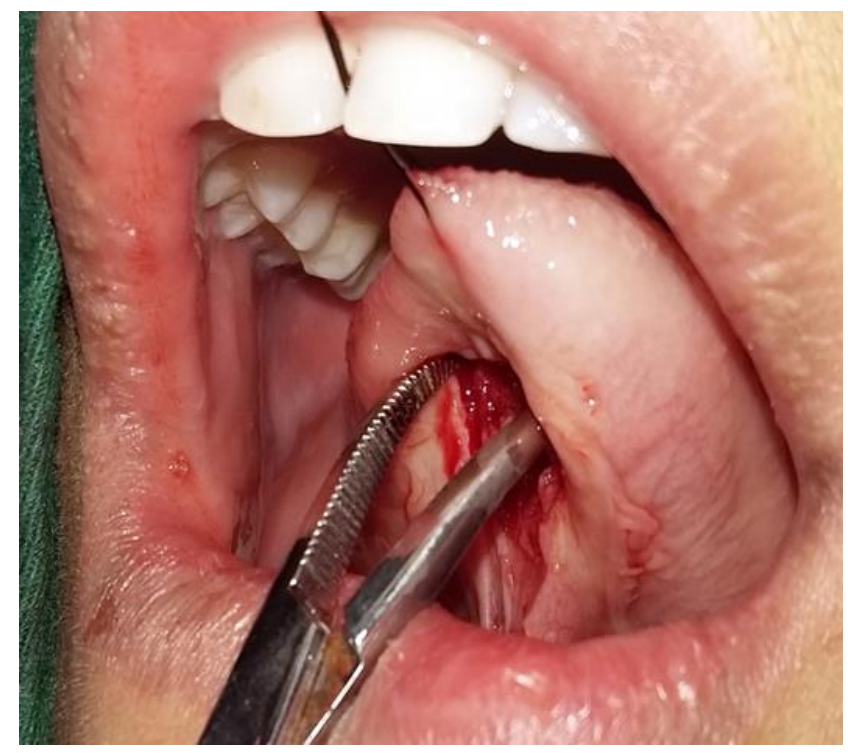

\author{
Imagen 5 Liberación De Fibras Musculares \\ Fuente: Registro De La Investigación \\ Autor: Thalía Salgado Rosado
}




\section{Tratamiento quirúrgico de la anquiloglosia recidivante: A propósito de un caso}

Vol. 1, núm. 4., (2017)

Thalía Carolina Salgado Rosado, Juan Carlos Obando Piedra, Pablo Rafael Salgado Rosado, Walter Kiko Salgado

Para el cierre del defecto causado por la incisión, se efectuó puntos de sutura simple separados a lo largo de la incisión longitudinal, sin tensión, y con hilo de sutura quirúrgico de seda 0.3, para que los tejidos puedan sanar por primera intención y de esa forma minimizar la formación de tejido cicatrizal. (IMAGEN 6)

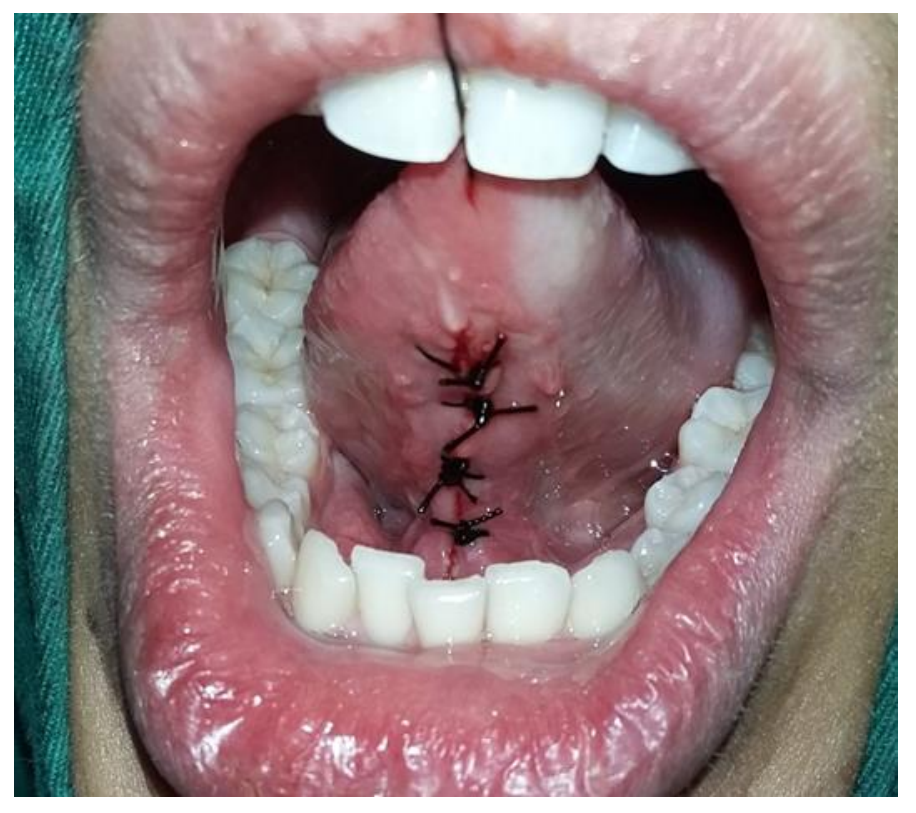

\section{Imagen 6 Finalizada la intervención quirúrgica Fuente: Registro De La Investigación Autor: Thalía Salgado Rosado,}

Una vez finalizada la intervención quirúrgica, se dio las indicaciones al paciente de los cuidados que debe tener durante su recuperación, como: colocarse hielo las primeras 24 horas postquirúrgicas para la inflamación y consumir alimentos blandos los primeros días Para prevenir infección posoperatoria y dolor, fueron prescritos Antiinflamatorios No Esteroides AINE: Tabletas de Ibuprofeno (VO) de $600 \mathrm{mg}$ cada 6 horas por 3 días, y Antibióticos: Capsula Amoxicilina (VO) de $250 \mathrm{mg}$ cada 8 horas por 7 días. 


\title{
Tratamiento quirúrgico de la anquiloglosia recidivante: A propósito de un
}

Vol. 1, núm. 4., (2017)

Thalía Carolina Salgado Rosado, Juan Carlos Obando Piedra, Pablo Rafael Salgado Rosado, Walter Kiko Salgado

El 1er control post operatorio se realizó a los 7 días de la frenectomía lingual, en la cual retiramos los puntos de sutura delicadamente, se observó cierre completo de la lesión y el sitio sin evidencia de infección. El segundo control lo realizamos a los 25 días de la cirugía, donde pudimos observar el excelente resultado del tratamiento; el paciente puede elevar la lengua sin dificultad al ser solicitado que contacto la lengua con el paladar, el paciente lo realiza sin problema además puede articular mejor las palabras, y existe satisfacción tanto del paciente como de los padres. (Imagen 7). La Imagen 8 muestra el tercer posoperatorio 1 año después.

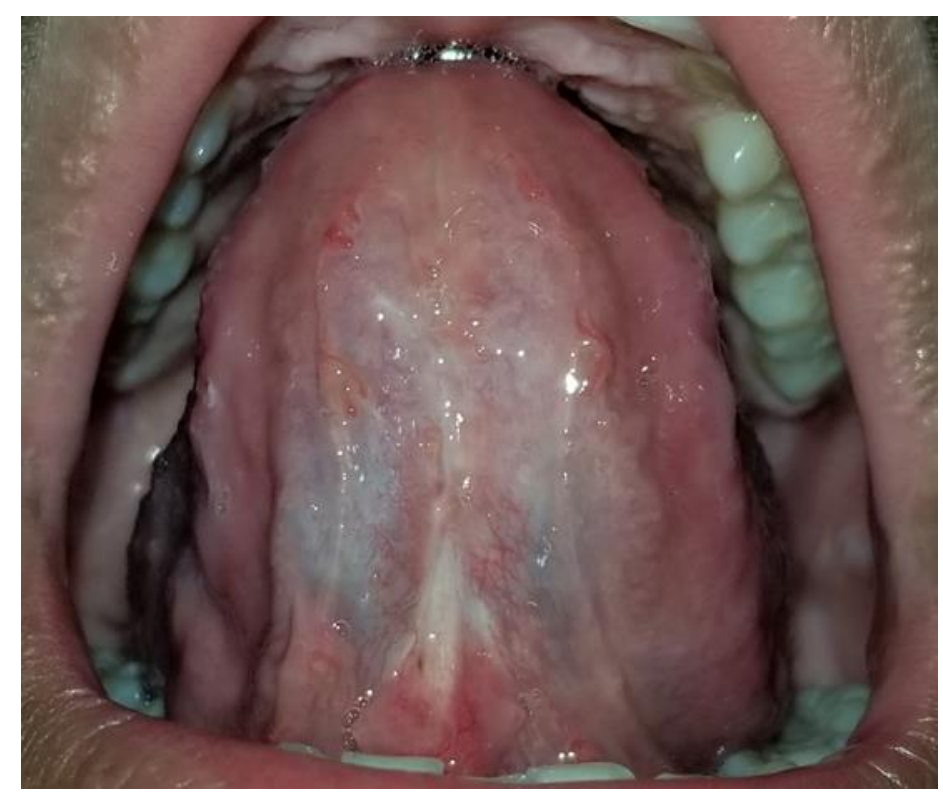

\author{
Imagen 7 Tercer Control Postoperatorio \\ Fuente: Registro De La Investigación \\ Autor: Thalía Salgado Rosado
}




\section{Tratamiento quirúrgico de la anquiloglosia recidivante: A propósito de un caso}

Vol. 1, núm. 4., (2017)

Thalía Carolina Salgado Rosado, Juan Carlos Obando Piedra, Pablo Rafael Salgado Rosado, Walter Kiko Salgado

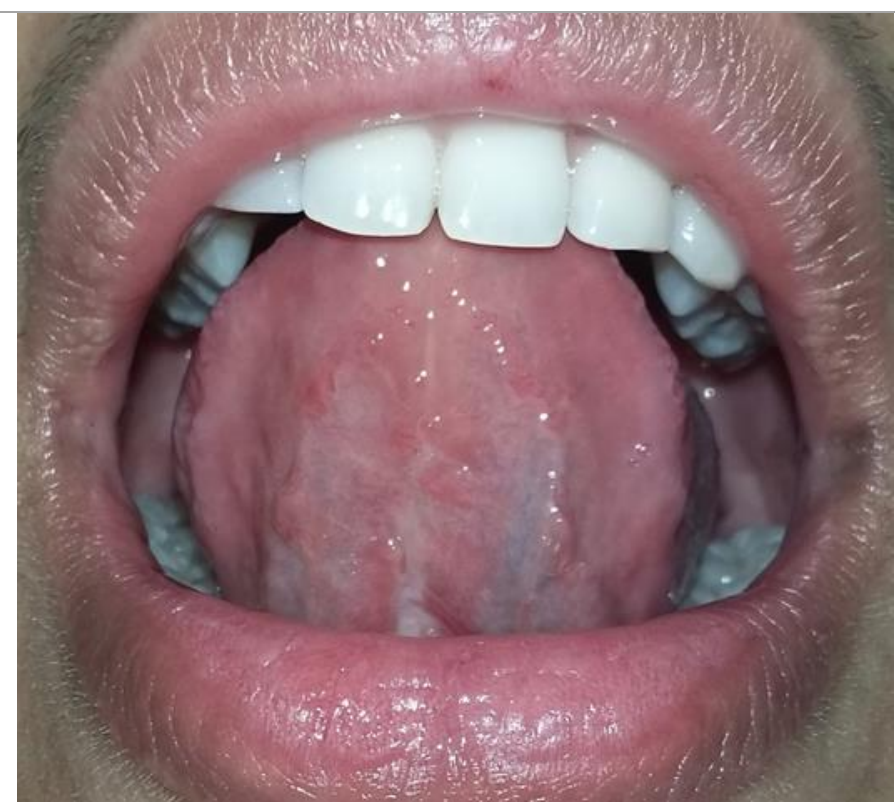

\section{Imagen 8 Segundo Control Postoperatorio \\ Fuente: Registro De La Investigación \\ Autor: Thalía Salgado Rosado}

\section{Discusión.}

La anquiloglosia es una patología congénita, pero no existen parámetros para su diagnóstico y tratamiento. Se han enumerado múltiples consecuencias de la anquiloglosia en neonatos e infantes, pero son pocos los estudios de las complicaciones a largo plazo de la anquiloglosia en el adulto. ${ }^{1-8}$

El caso clínico que presentamos utilizamos la técnica de frenectomía lingual con una pinza hemostática, los resultados obtenidos fueron satisfactorios, el paciente mejoro el movimiento lingual y optimizó la articulación de las palabras, se concluye que siempre que acompañemos la frenectomía con rehabilitación miofuncional. ${ }^{8-13}$ 


\section{Tratamiento quirúrgico de la anquiloglosia recidivante: A propósito de un}

Vol. 1, núm. 4., (2017)

Thalía Carolina Salgado Rosado, Juan Carlos Obando Piedra, Pablo Rafael Salgado Rosado, Walter Kiko Salgado

Concordamos con el estudio que demuestra que la técnica quirúrgica frenectomía, mejora en diferentes grados, la postura de la lengua, la movilidad de la lengua, las funciones orofaciales, la postura del labio inferior, independientemente de que tipo de rehabilitación oral reciba. Es prueba de que la frenectomía debería ser referida en la mayoría de casos de anquiloglosia. ${ }^{10-13}$

Discrepamos con el estudio que sugiere que la tasa de los casos de frenillo lingual recidivante es elevada cuando son realizados sin anestesia general y en una oficina dental. En el presente trabajo si encontramos diferencia entre la tasa de recidiva de la técnica quirúrgica frenotomía y la frenectomía, y proponemos que toda división de frenillo lingual debería ser bajo anestesia local para su éxito. ${ }^{13}$

\section{Conclusiones.}

La Anquiloglosia recidivante que presenta el paciente es el resultado de una frenotomía lingual fallida realizada en sus primero años de vida, sin la respectiva rehabilitación miofuncional.

El protocolo puesto en práctica, de la técnica quirúrgica de frenectomía lingual acompañada de ejercicios miofuncionales ejecutados antes, y después de la cirugía, obtuvo resultados satisfactorios para la rehabilitación del paciente con anquiloglosia.

El paciente se mostró satisfecho y logro el movimiento de la lengua libremente, lo cual mejoro notablemente su problema con la articulación de las palabras. 


\section{Tratamiento quirúrgico de la anquiloglosia recidivante: A propósito de un caso \\ Vol. 1, núm. 4., (2017) \\ Thalía Carolina Salgado Rosado, Juan Carlos Obando Piedra, Pablo Rafael Salgado Rosado, Walter Kiko Salgado}

\section{Bibliografía.}

1. Ferrés E, Pastor T, Ferrés E, Mareque J, Prats J, \& Padró E. Multidisciplinary management of ankyloglossia in childhood. Treatment of 101 cases. A protocol. Pediatric Dentistry. 2016; 21(1): 39-47.

2. Azevedo M, Oliveira N, Costa e Silva J, Borges L, Silveira A, Gomes C, \& Thiemy V. Surgical techniques for the treatment of ankyloglossia in children: a case series. J Appl Oral Sci. 2014; 22(3); 241-248.

3. Gay C., \& Berini L. Tratado de cirugía bucal Vol. 1. 2014. Ergon: Barcelona.

4. Amir L, James J, \& Donath S. Rehability of the Hazelbaker Assessment Tool for Lingual Frenulum Function. International Breastfeeding Journal. 2006; 1(3): 3-9.

5. Moore K, \& Dalley A. Anatomía con orientación clínica (5ta edición). 2007; Madrid: Editorial Panamericana.

6. Philip J, Reversole L, \& Wysocki G. Patología oral y maxilofacial contemporánea ( $2^{\text {da }}$ edicion ed.). 2004. Madrid: Elsevier.

7. Klockars T, \& Pitkäranta A. Pediatric tongue-tie division: Indications, techniques and patient satisfaction. Int J Pediatr Otorhinolaryngol. 2009 Oct; 73(10): 1399-401.

8. Kotlow L. Laser in pediatric dentistry. Alpha Omegan. 2008; 101(3): 889-922.

9. Kotlow L. Lasers and Soft Tissue Treatments for the Pediatric Dental Patient. Alpha Omegan. 2006; 101(1): 143-146.

10. Langlais R, Miller C, \& Nield-Gehrig J. Atlas a color de enfermedades bucales. 2014. México: El Manual moderno.

11. Marchese I, Lopes de Castro C, \& Jordão R. Lingual frenulum: changes after frenectomy. 2012; 13(1): 409-412.

12. Williamn WN, \& Waldron CM. Assessment of lingual function when anquiloglosia (Tongue-tie) is suspected. J. Am Dent Assoc. 1985; 110(1): 353-356.

13. Yousefi J, Tabrizian F, Mohammad S, Gillies R, Ashkezari A, \& Meara J. Iran J. Tongue-tie Repair: Z-Plasty Vs Simple Release. Otorhinolaryngol. 2015 Mar; 27(79): $127-135$. 